\section{Plunging ranula in children: case report and literature review}

\author{
Veronica Carlini,1 Valeria Calcaterra,2 \\ Noemi Pasqua, 1 Marinella Guazzotti, ${ }^{1}$ \\ Mario Fusillo, 1 Gloria Pelizzo 3 \\ 1Pediatric Surgery Unit, Department of \\ Maternal and Children's Health, \\ Fondazione IRCCS Policlinico S.Matteo, \\ Pavia, Italy; 2Pediatric Unit, Department \\ of Maternal and Children's Health, \\ Fondazione IRCCS Policlinico S.Matteo \\ and Department of Internal Medicine and \\ Therapeutics, University of Pavia, Pavia, \\ Italy; 3Pediatric Surgery Unit, \\ Department of Maternal and Children's \\ Health, Fondazione IRCCS Policlinico \\ S.Matteo and Department of Clinical, \\ Surgical, Diagnostic and Pediatric \\ Sciences, University of Pavia, Pavia, and \\ Ospedale dei Bambini "G. Di Cristina”, \\ A.R.N.A.S. Palermo, Italy
}

\section{Abstract}

Few cases of plunging ranulas (PRs) occur during childhood and the lesions are frequently misdiagnosed. Here, a PR in a child is reported along with a literature review. A seven-year-old female complaining of swelling in the midline neck, left-submandibular region, was evaluated. No oral cavity or major salivary glands abnormalities were detected. On palpation, a soft, painless, and fluid-containing mass was observed. The suspicion PR was performed by ultrasound. The diagnosis was confirmed with a histopathological examination. The lesion was removed with a cervical approach, without recurrence. PR is an uncommon condition in children under 10 years of age. Differential diagnosis depends on clinical examination and ultrasonography. A computed tomography-scan and magnetic resonance imaging can be performed if the diagnosis remains uncertain. In pediatrics, the key to success of the treatment may rely on the radical excision of the cyst and sublingual gland, via an intraoral or submandibular approach.

\section{Introduction}

The ranula is a mucus-filled cyst like mass that usually originates from extravasation of mucus from the sublingual gland (intraoral ranula). The ranula is referred to as a plunging ranula (PR) when it affects the submandibu- lary space and adjacent structures in the neck. ${ }^{1-3}$ PR is an uncommon condition, which presents itself as a fluctuating swelling in the neck. It most frequently occurs in patients under the age of 30 years, and rarely in young children. 4

The etiology of PR is still unknown but, an association with trauma, congenital anomalies and sublingual glandular diseases have been described. 3,5 It is accepted that ranula formation is thought to be secondary to a blockage of the sublingual gland ducts, which leads to backpressure of mucin and subsequent extravasation of mucus, through a hiatus in the mylohyoid muscle. ${ }^{3,5}$

PR is usually centered in the submandibular area, but can extend into the submental space, both cervical areas, the retropharyngeal space or the supraclavicular area. It generally appear in conjunction with an oral ranula and rarely do they arise independently of an oral component. 5 When there is no clinical evidence of an oral association, diagnostic acumen is necessary. It is sometimes mistaken for a thyroglossal duct cyst, lymphadenopathy, abscess, dermoid cyst, glottic cyst or thyroid cyst.1,5 Advanced diagnostic techniques like ultrasound, computed tomography (CT) and magnetic resonance imaging (MRI) plays an important role in the diagnosis. ${ }^{5-8}$

Surgical and non-surgical methods are used to treat ranula, however the debate on the optimal PR treatment in pediatrics is still open. $5,9,10$

A case of a PR without oral swelling in a seven-year-old female is discussed along with a literature review relative to this type of the lesion in young children.

\section{Case Report}

A seven-year-old Caucasian female visited our Pediatric Surgery Unit complaining of swelling in the midline neck, left-submandibular region. The swelling had a sudden onset two weeks earlier. No abnormality of the floor of the mouth, jaws, teeth or major salivary glands was detected. On palpation, the exam revealed a soft, painless, and fluid-containing mass. The rest of soft tissue planes and mandible appeared normal with no evidence of destruction. The patient was in good health and had no history of any systemic disorder. No trauma or surgical history were reported. The swelling did not cause difficulty in swallowing or speaking. Routine blood tests and the thyroid profile were within normal limits.

Ultrasonography revealed an ovoidal neoformation in the submandibular triangle, measuring $2 \times 2 \mathrm{~cm}$; the echostructuture was mixed, partly liquid and partly solid. Color Doppler did not reveal internal vascularization. A provi-
Correspondence: Gloria Pelizzo, Pediatric Surgery Unit, Ospedale dei Bambini "G. Di Cristina" Via dei Benedettini 1, 90134 Palermo, Italy. Tel. +091-6666007.

E-mail: gloriapelizzo@gmail.com

Key words: Plunging ranula; Children; Differential diagnosis; Therapy.

Acknowledgments: the authors would thank Dr. L. Kelly for English revision of the manuscript.

Contributions: VeC collected the patient data, performed surgical treatment and drafted the manuscript. VaC drafted the manuscript and revised the literature. NP helped to draft the manuscript. MG performed ultrasonography and helped to draft the manuscript. MF helped to draft the manuscript. GP critically revised the manuscript.

Conflict of interest: the authors declare no potential conflict of interest.

Received for publication: 2 May 2016.

Accepted for publication: 4 October 2016.

This work is licensed under a Creative Commons Attribution NonCommercial 4.0 License (CC BYNC 4.0).

CC Copyright V. Carlini et al., 2016

Licensee PAGEPress, Italy

Pediatric Reports 2016; 8:6576

doi:10.4081/pr.2016.6576

sional diagnosis of PR was made. However, a tyroglossal duct cyst was not excluded, because of its position in the midline of the neck, even if vertical movement with tongue protrusion and swallowing were not evident.

Surgical excision of the mass was carried out under general anesthesia. During surgery, aspiration was carried out to confirm that the cystic mass contained thick straw-colored mucus. It extended into the sublingual space by crossing over the posterior border of mylohyoid muscle. The lesion was not connected to the foramen cecum or the central portion of the hyoid bone. The diagnosis of plunging ranula was made. The sublingual glandular tissue, to which the cyst was adherent, was totally removed by a cervical approach. The salivary contents of the cyst were evacuated and the cyst wall was resected. A drainage strip was inserted in place of the cyst and removed on postoperative day 2. A pressure dressing was applied to the submandibular region for at least two days. Histopathological examination confirmed sublingual glandular tissue with mild chronic inflammation (fibroblastic reaction, connective tissue and saliva). Eightmonths post operatively the patient has not experienced any recurrence. 


\section{Discussion}

PRs are rare cystic masses of the neck that are essentially mucous retention pseudocysts from an obstructed sublingual gland. They plunge by extending inferiorly beyond the free edge of the mylohyoid muscle, or through a dehiscence of the muscle itself, to enter the submandibular space.

The prevalence of PRs is unknown, but it is thought to be low.1-4,6 There are several hundred reported cases of PR in the literature. Although the majority of these have occurred in patients in the second or third decade of life, a few cases have been reported in children less than 10 years of age. ${ }^{4,11}$

PR occurs as the result of trauma or infection which in turn causes direct damage to the duct or deeper areas of the sublingual gland. $3,5,6$ A congenital predisposition, particularly in children has been suggested, given the predominance of ranulas in young siblings. ${ }^{11}$ The report of PR in a one- year-old child supports a possible congenital origin.11

PRs form only in the sublingual gland because it is the only salivary gland that secretes continuously, even during the interdigestive period. The high protein content in the secretions is believed to produce a very intense inflammatory reaction and may mediate pseudocyst formation.

The PR may arise in the neck by four possible mechanisms (Figure 1).2,5 Firstly, the sublingual gland may project through the mylohyoid to provide an origin. Secondly the cyst may penetrate through the mylohyoid to join the sublingual gland in the mouth. Thirdly, up to $44 \%$ of PRs occur iatrogenically after surgery to remove oral ranulas. Lastly, a duct from the sublingual gland may join the submaxillary duct and give rise to a ranula in continuity with the submandibular gland or its duct. The latter explanation is favored as the ranula was adherent to the submaxillary gland, but recurred after the gland was excised.1,5

The diagnosis of a PR is usually determined by a combination of history, clinical presentation, and imaging studies (Table 1). Due to its low incidence and its variegated clinical presentation, PR is a frequently misdiagnosed condition. ${ }^{5}$

Clinically, a PR presents as a painless, fluctuant, gradually enlarging lateral neck swelling which does not usually change with swallowing or eating. It is most commonly centered on the submandibular triangle and averages 4-10 $\mathrm{cm}$ in size. It can extend superiorly in to the parapharyngeal space as far as the skull base, inferiorly to the supraclavicular area, posteriorly into the retropharyngeal space or across the midline anteriorly. PRs generally appear in conjunction with an oral ranula; but at least $20 \%$ can arise independently of an oral component. ${ }^{5,9}$ In the absence of oral swelling, the clinical diagnosis of ranula may not be suspected and a differential diagnosis may be difficult. PRs should be considered in the differential diagnosis of thyroglossal duct cyst, intramuscular hemangioma, lipomas, cystic/neoplastic thyroid disease, branchial cyst, submandibular sialadenitis, laryngocele, dermoid cyst, lymphatic or vascular malformations and infectious cervical lymphadenopathy like tuberculosis, Epstein-Barr virus, cervical thymic cysts, dermoid cysts, cystic hygroma and benign teratoma.3,5

These entities cannot be distinguished one from another by clinical evaluation alone, so

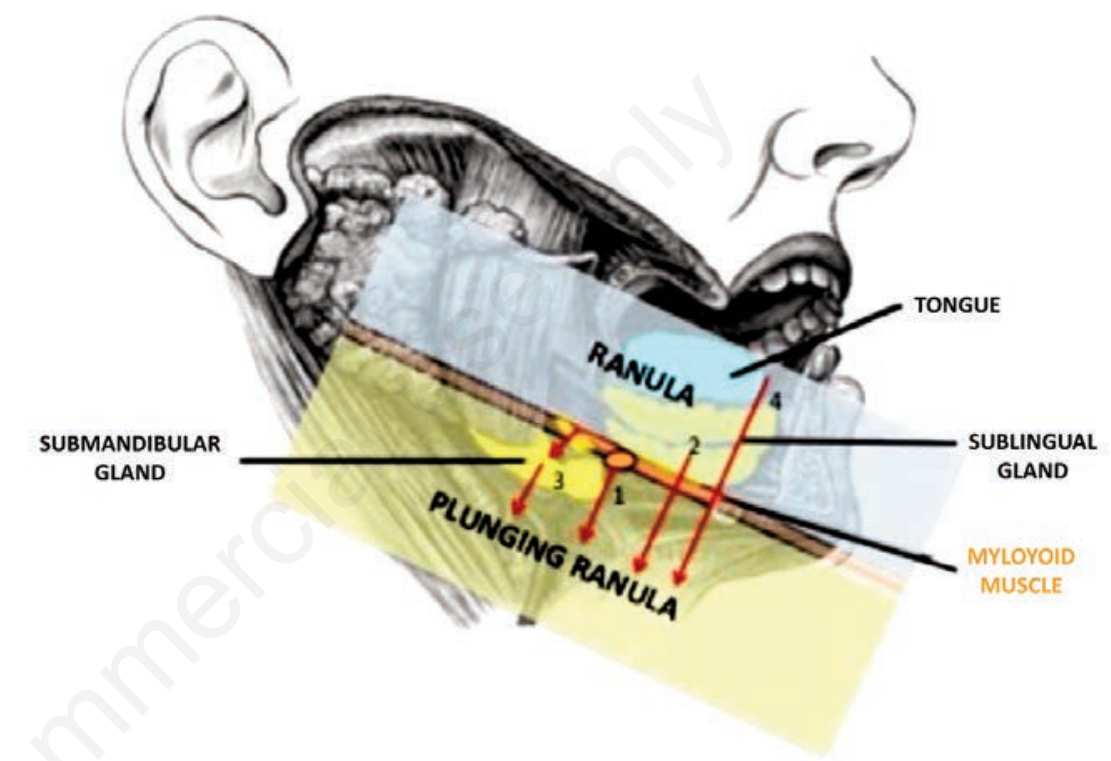

Figure 1. Pathogenetic mechanisms of plunging ranula development: 1) the sublingual gland may project through the mylohyoid to provide an origin; 2 ) the cyst may penetrate through the mylohyoid to join the sublingual gland in the mouth; 3) a duct from the sublingual gland may join the submaxillary duct and give rise to a ranula in continuity with the submandibular gland or its duct; 4) plunging ranulas may occur iatrogenically after surgery to remove oral ranulas.

Table 1. Elements used to diagnose plunging ranula.

\begin{tabular}{ll}
$\begin{array}{l}\text { Origin } \\
\text { Clinical signs }\end{array}$ & $\begin{array}{l}\text { Sublingual glands; sublingual gland in continuity with the submandibular gland. } \\
\text { Lateral neck swelling, commonly centered on the submandibular triangle; possible expansion superiorly into the } \\
\text { parapharyngeal space, inferiorly to the supraclavicular area, posteriorly into the retropharyngeal space or across } \\
\text { the midline anteriorly; } 80 \% \text { conjunction with an oral ranula; } 20 \% \text { without an oral component. }\end{array}$ \\
\hline Differential diagnosis & $\begin{array}{l}\text { Thyroglossal duct cyst, intramuscular hemangioma, lipomas, cystic/neoplastic thyroid disease, branchial cyst, } \\
\text { submandibular sialadenitis, laryngocele, dermoid cyst, lymphatic or vascular malformations, infectious cervical ly } \\
\text { phadenopathy, thymic cysts, dermoid cysts, cystic hygroma, benign teratoma. }\end{array}$ \\
Imaging & $\begin{array}{l}\text { Altrasonography: cystic lesion (ovoidal or loblulated) in the submandibolar triangle. } \\
\text { Computed tomography: unilocular cystic lesion with thin or imperceptible walls and homogeneous intracystic }\end{array}$ \\
& $\begin{array}{l}\text { low attenuation similar to other simple fluid-containing structures. } \\
\text { Magnetic resonance imaging: thin-walled cystic structure with low to intermediate signal on T1-weighted sequences } \\
\text { and high signal on T2-weighted images. }\end{array}$ \\
\hline Fine needle aspiration cytology & $\begin{array}{l}\text { Yellow aspirate, positive amylase and mucin. No presence of epithelial/glandular elements, cholesterol crystal and keratin. } \\
\text { Therapy }\end{array}$ \\
Conservative treatment: sclerotherapy, cryotherapy. \\
Surgical treatment: intraoral approach, submandibular approach.
\end{tabular}


diagnosis relies on imaging or fluid aspiration from the cervical swelling.

There is no consensus on the optimal imaging modality to detect plunging ranula. Ultrasonography is one of the best tools and is reliable in order to identify the presence of cystic lesions in the submandibular triangle as well as a mylohyoid defect, which is associated with this condition in up to $90 \%$ of cases. Jain and colleagues recommended an ultrasonography for pediatric ranula diagnosis, as it does not necessitate sedation or radiation exposure.7,8 Jain and colleagues have been performing ultrasonography for cysts for 10 years; 7,8 real-time, high-resolution ultrasound can clearly identify active herniation of the sublingual gland and can measure the extent of cervical space involvement with the pseudocyst for the plunging ranula.

As in our case, ultrasound often depict an ovoidal or loblulated cystic lesion, or anaecogena or h low-level internal echoes. Color Doppler investigation reveals no intracystic vascularity and none to minimal peripheral hyperemia. 8 Whenever the diagnosis remains unclear after clinical assessment and ultrasound imaging, CT scan and MRI can be performed as a complement.5,7 With CT scans, ranulas are usually unilocular cystic lesions with thin or imperceptible walls and homogeneous intracystic low attenuation similar to other simple fluid-containig structures.6,7,9,12 MRI shows a thin-walled cystic structure with a low to intermediate signal on T1-weighted sequences and a high signal on T2-weighted images. Coit and colleagues conclude that the diagnosis of PR is strongly suggested by the tail sign in MRI, a slight extension into the sublingual space with the bulk of the cyst seen in the submandibular space. 12 Aside from clinical and imaging assessments, diagnosis is definitively established with fine needle aspiration cytology (FNAC) and the presence of yellow aspirate, positive amylase and mucin, but devoid of epithelial/glandular elements, cholesterol crystals and keratin.,3 Although FNAC cannot be performed under local anesthetic in all children, the information obtained is diagnostic and justifies performing the procedure under general anesthesia. ${ }^{3}$ PR rarely resolves spontaneously. Several methods of treatment for PRs have been reported, including sclerotherapy, marsupialization, excision of the pseudocyst, and excision of the sublingual and/or submandibular gland.5,10,13 To date, it remains unclear which is the most appropriate treatment procedure in children. Sclerotherapy with $0 \mathrm{OK}-432$ is commonly recommended as a well tolerated and effective primary treatment for ranulas also in pediatric patients. 14 The mechanism by which $0 \mathrm{~K}-432$ acts in PR may be by increasing absorbption and decreasing saliva production caused by local inflammatory reactions, resulting in the collapse and adhesion of the pseudocyst. However, sclerotherapy of PR in children is rare. The effectiveness of $0 \mathrm{~K}-432$ could be affected by factors such as the initial size of the lesion and the number and interval of injections. ${ }^{14} \mathrm{Kim}$ and colleagues revealed that only $66.7 \%$ of PR showed a marked response after one to three $0 \mathrm{~K}-432$ injections (injection every 15 days), with a recurrence rate of 12.5\%.14 Cryotherapy after marsupialization has also been suggested. ${ }^{15}$ In our opinion, as well as other authors, cryosurgical procedures are not satisfactory because the effect of freezing on the submandibular duct is also unknown. Furthermore, this technique may cause potential injury to the lingual nerve and to the submandibular duct. To date, surgery is the preferred method to treat PR. Marsupialization and excision of the pseudocyst with or without removal of the submandibular gland almost always leads to recurrence of PR. The risk of recurrence is greater than $50 \%$ and it depends on complete sublingual gland excision and the induction of sufficient fibrosis to seal the mucus extravasation. ${ }^{5}$

Consequently, as adopted in our case, the most efficacious treatment is now considered to be surgical excision of the ipsilateral sublingual gland. ${ }^{5}$ Surgical treatment of PR in pediatrics is often more difficult than in adults because of its smaller size, thinner wall, and greater friability. Excision via an intraoral approach is preferred in adult patients, although management via a submandibular incision with PR excision along with the sublingual gland has also been described.3,15 This approach should be useful in case of uncertain diagnosis, in order to obtain a better exploration of the neck region. The submandibular incision carries the potential risk of injury to the marginal mandibular, lingual, and hypoglossal nerve and the risk of cervical fistula formation, as well as cervical scarring. On the other hand, the sublingual gland is the source of the problem, so it is unnecessary to remove the pseudocyst via a neck incision. Closing the partial dehiscence of the mylohyoid is not regained if the entire ipsilateral sublingual gland is removed. Moreover leaving the mylohyoid dehiscence open helps improve the drainage of saliva or blood from the pseudocyst of the neck in the postoperative period. ${ }^{5}$

\section{Conclusions}

PR is an uncommon condition in young children. The PR without an oral component is frequently misdiagnosed. The differential diagnosis depends on clinical examination and ultrasonography. Complementary CT scan and MRI can be performed if the diagnosis remains uncertain. Although the treatment of PR is still controversial, the key to success remains the radical excision of the cyst and ipsilateral sublingual gland, via an intraoral or submandibular approach, with low morbidity and absence of recurrence.

\section{References}

1. de Visscher JGAM, van der Wal KGH, de Vogel PL. The plunging ranula. Pathogenesis, diagnosis and management. J Craniomaxillofac Surg 1989;17: 182-5.

2. Venkat Suresh B, Sambhav K. Vora huge plunging ranula. J Maxillofac Oral Surg 2012;11:487-90.

3. Mahadevan M, Vasan N. Management of pediatric plunging ranula. Int $\mathrm{J}$ Pediatr Otorhinolaryngol 2006;70:1049-54.

4. Mizuno A, Yamaguchi K. The plunging ranula. Int $\mathbf{J}$ Oral Maxillofac Surg 1993:22:113-5.

5. Zhi K, Gao L, Ren W. What is new in management of pediatric ranula? Curr Opin Otolaryngol Head Neck Surg 2014;22:5259.

6. 0'Connor R, Mc Gurk M. The plunging ranula: diagnostic difficulties and a less invasive approach to treatment. Int $\mathrm{J}$ Oral Maxillofac Surg 2013;42:1469-74.

7. Jain R, Morton RP, Ahmad Z. Diagnostic difficulties of plunging ranula: case series. J Laryngol Otol 2012;126:506-10.

8. Jain P, Jain R, Morton RP, Ahmad Z. Plunging ranulas: high-resolution ultrasound for diagnosis and surgical management. Eur Radiol 2010;20: 1442-9.

9. Zhi K, Gao L, Ren W. What is new in a management of pediatric ranula? Curr Opin Otolaryngol Head Neck Surg 2014;22:5259.

10. Haberal I, Gocmen H, Samim E. Surgical management of pediatric ranula. Int $\mathrm{J}$ Pediatr Otorhinolaryngol 2004;68:161-3.

11. Matt BH, Crockett DM. Plunging ranula in an infant. Otolaryngol Head Neck Surg 1988;99:330-3.

12. Coit WE, Harnsberger HR, Osborn AG, et al. Ranulas and their mimics: CT evaluation. Radiology 1987;163:211-6.

13. Sigismund PE, Bozzato A, Schumann M, et al. Management of ranula: 9 years' clinical experience in pediatric and adult patients. J Oral Maxillofac Surg 2013;71:538-44.

14. Kim KH, SungMW, RohJL, HanMH. Sclerotherapy for congenital lesions in the head and neck. Otolaryngol Head Neck Surg 2004;131:307-16.

15. Yang Y, Hong K. Surgical results of the intraoral approach for plunging ranula. Acta Otolaryngol 2014;134:201-5. 